\title{
Composition of Wave Modes in Magnetosheath Turbulence from Sub-ion to Sub-electron Scales
}

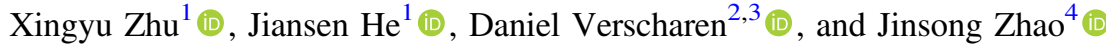 \\ ${ }^{1}$ School of Earth And Space Sciences, Peking University, Beijing, 100871, People’s Republic of China; jshept@pku.edu.cn \\ ${ }^{2}$ Mullard Space Science Laboratory, University College London, Dorking RH5 6NT, UK \\ ${ }^{3}$ Space Science Center, University of New Hampshire, Durham, NH 03824, USA \\ ${ }^{4}$ Purple Mountain Observatory, Chinese Academy of Sciences, Nanjing, 210008, People's Republic of China \\ Received 2018 December 12; revised 2019 March 24; accepted 2019 April 22; published 2019 June 12
}

\begin{abstract}
The nature of plasma turbulence remains unclear at kinetic scales, especially sub-electron scales. We simultaneously investigate spectra of magnetic and electric fields from proton scales $(\sim 1 \mathrm{~Hz})$ down to subelectron scales $(\sim 1 \mathrm{kHz})$ using $M M S$ measurements in the magnetosheath turbulence. We find that magnetic field spectra exhibit identical behavior between ion and electron scales, while they bifurcate at about $30 \mathrm{~Hz}$ (around electron inertial scale) and then aggregate around $1 \mathrm{kHz}$ (around electron gyrofrequency). Kinetic Alfvén waves (KAWs) are identified in the range from 1 to $30 \mathrm{~Hz}$ : (1) the power spectral indices of $\operatorname{PSD}(B)$ and $\operatorname{PSD}(E)$ are about -3 and -1 ; (2) parallel magnetic and electric fluctuations are present but subdominant. Magnetic field spectra obtained from postnoon region events show a clear spectral enhancement, which is identified as quasi-parallel whistler waves (WWs). Electric field spectra present a wide bulge around $800 \mathrm{~Hz}$, which is also seen as island-like patterns in the time-period wavelet spectra. The electric field spectral enhancement is dominated by field-parallel fluctuations and is, therefore, diagnosed as ion acoustic waves (IAWs). We also perform a quantitative analysis of the multiple mode types using polarization predictions from two-fluid theory. We obtain the energy partition of electromagnetic fields for different wave modes and successfully reproduce the observed PSDs of $B_{\perp}, B_{\|}, E_{\perp}$, and $E_{\|}$. Our findings suggest that the magnetosheath turbulence consists of a mixture of multiple wave modes (KAW, WW, and IAW) in the range from sub-ion scales to sub-electron scales.
\end{abstract}

Key words: turbulence - waves

\section{Introduction}

Turbulence exists in almost all heliospheric plasma environments like the solar wind (e.g., Tu \& Marsch 1995; Alexandrova et al. 2013; Bruno \& Carbone 2013), the magnetosheath (e.g., Sahraoui 2003; He et al. 2011; Huang et al. 2014), and the solar atmosphere (e.g., Petrosyan et al. 2010; Hollweg et al. 2013). The magnetosheath is the plasma region downstream Earth's bow shock, which is generated by the interaction of the supersonic solar wind and the Earth's magnetosphere. Compared to the upstream solar wind, the magnetosheath is characterized by a greater magnitude of the magnetic field, plasma density, and temperature.

We identify four characteristic regimes in plasma turbulence: the energy-containing range, the inertial range, the electronfluid inertial range/ion-kinetic dissipation range, and the fully kinetic dissipation range. In the inertial range, non-compressive magnetic fluctuations are usually attributed to Alfvénic fluctuations, while compressive fluctuations may correspond to propagating slow magnetosonic waves, fast magnetosonic waves, or convective pressure-balanced structures. In the solar wind, especially in fast streams, non-compressive Alfvénic turbulence is a remarkably universal phenomenon (Belcher \& Davis 1971; Tu \& Marsch 1995). Alfvénic turbulence displays a power spectrum in accordance with Kolmogorov's theory (Kolmogorov 1941) with a spectral index of approximately $-5 / 3$. The compressive fluctuations have been found more likely to be pressure-balanced structures with polarizations like oblique slow magnetosonic waves rather than fast magnetosonic waves (Tu \& Marsch 1995; Yao et al. 2011; Howes et al. 2012; He et al. 2015; Yang et al. 2017). Magnetosheath turbulence is predominantly compressive and shows a flattened magnetic field power spectrum, attributed to the compression of the solar wind at the bow shock (Narita et al. 2016; Huang et al. 2017).

When the cascading turbulent energy reaches ion scales and beyond, the turbulence becomes dispersive and dissipative. The nature of turbulence at kinetic scales is still under intense debate. Statistical research of solar wind streams with different speed reveals that magnetic spectra have different power levels in the inertial range while they converge toward one profile at ion scales (Bruno et al. 2014). From the ion scales down to the electron scales, the spectrum of magnetic fluctuations is a power law with a power index close to -2.8 based on observations both in the solar wind (Alexandrova et al. 2009; Sahraoui et al. 2013) and in the magnetosheath (Huang et al. 2014). Kinetic Alfvén waves (Bale et al. 2005; Sahraoui et al. 2010) and oblique whistler/ Bernstein waves (Stawicki et al. 2001; Perschke et al. 2013) are suggested as candidates for the fluctuations below the proton gyroscale. At sub-electron scales, the morphology of the spectrum is still controversial. Sahraoui et al. (2010) report that the spectrum below electron scales continues as a power-law spectrum. On the other hand, Alexandrova et al. (2012) describe the spectrum below electron scales as an exponential function with a power-law factor, which may be a result of Landau damping at electron scales. Recently, Chen \& Boldyrev (2017) suggest that inertial kinetic Alfvén waves (IKAWs) may be active on scales smaller than the electron inertial length in an event, in which $\beta_{i} \sim 0.8$ and $\beta_{e} \sim 0.08$, where $\beta_{s}=2 \mu_{0} n_{s} k_{\mathrm{B}} T_{s} / B_{0}^{2}, \mu_{0}$ is the vacuum permeability, $n_{s}$ is the background density of species $s, T_{s}$ is the temperature of species $s, B_{0}$ is the background magnetic field, and $k_{\mathrm{B}}$ is the Boltzmann constant. They identify a 
transition from kinetic Alfvén waves at $k d_{e}<1$ to inertial kinetic Alfvén waves at $k d_{e}>1$, where $d_{e}$ is the electron inertial length. The magnetic power spectrum for IKAW-turbulence is predicted to be steep with a power-law index of $-11 / 3$. We address the question of whether IKAW are the only mode type of magnetosheath turbulence at scales beyond the electron inertial length or whether other types of waves (e.g., whistler waves) contribute.

Compared to the magnetic field power spectral density (PSD), the electric field PSD presents a different trend from fluid scales to kinetic scales. At fluid scales, the electric spectrum follows directly from the velocity spectrum due to the Lorentz transformation from the plasma frame (Chen et al. 2011). Around and beyond proton scales (inertial length and thermal gyroradius), the electric field PSD flattens, associated with a monotonic increase of the electric-to-magnetic PSD ratio (Bale et al. 2005; Sahraoui et al. 2009). In the magnetosheath, electrostatic waves are occasionally observed, some of which exhibit features consistent with solitary waves (Rodriguez \& Gurnett 1975; Gallagher 1985; Graham et al. 2016). These waves cause a unique feature with wide-band festoon-shaped emission in frequency-time spectrograms (Gallagher 1985) and a maximum electric fluctuation power at $\sim 1 \mathrm{kHz}$ above the ion plasma frequency $f_{p i}$ and below the electron plasma frequency $f_{p e}$ (Graham et al. 2016).

There are two kinetic instabilities that generate parallelpropagating whistler waves. If the electron distribution function exhibits a large enough anisotropy with $T_{e, \|}<T_{e, \perp}$, the plasma drives the whistler instability (Gary \& Wang 1996). Another instability driving whistler waves is the heat flux instability (Gary et al. 1999; Fujimoto 2014; Lacombe et al. 2014), which is excited if the electron heat flux $Q_{e}$ exceeds some threshold value. For the whistler waves observed in the magnetosheath, however, it remains unclear as to whether one of these linear instabilities is responsible for the generation of the observed whistler waves. Alternatively, a nonlinear and/or nonlocal process may be the source for whistler waves in this regime.

The ion acoustic wave (IAW) is a compressive electrostatic wave mode. Also IAWs can be driven by linear instabilities, for example, through the electron-ion drift instability (Fried \& Gould 1961) or the ion beam instability (Fried \& Wong 1966). These instabilities are essentially excited if the distribution function exhibits a positive derivative in velocity space at the parallel phase speed of the wave. The observations of broadband electrostatic noise (Scarf et al. 1974; Gurnett et al. 1976) are interpreted as a consequence of the ion beam instability (Gary 1993). The festoon-shaped emissions in the magnetosheath are speculated to be ion acoustic waves, generated upstream of the bow shock and then convected downstream (Gallagher 1985). We address the possibility of coexisting electrostatic IAWs with other types of electromagnetic waves in the kinetic regime from sub-ion to sub-electron scales in this work.

The nature of magnetosheath turbulence in the full wavenumber range from ion scales down to sub-electron scales is an important research topic that we address in this work. In the low- $\beta(\ll 1)$ magnetosheath, magnetic energy dominates through MHD scales and into ion-kinetic scales, while electron-kinetic energy exceeds magnetic energy below the electron inertial length (Gershman et al. 2018). Furthermore, using our theoretical model, we investigate the contributions of different wave modes (KAWs, WWs, IAWs) to the magnetic and electric field fluctuations from ion scales to

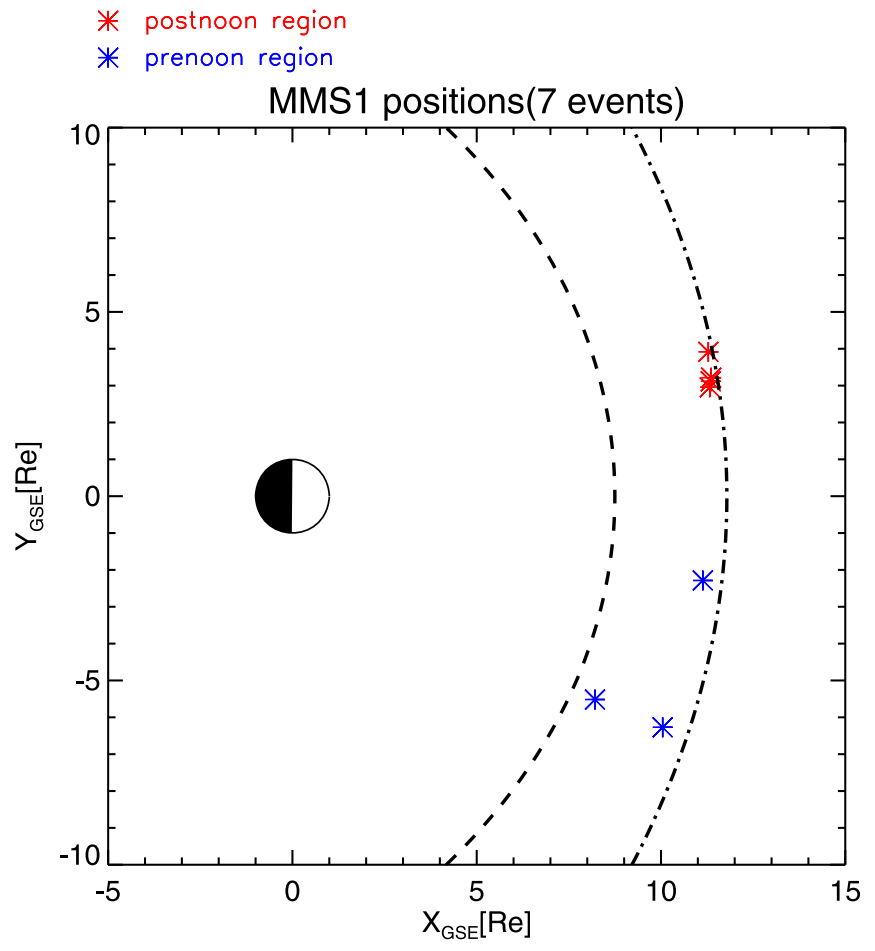

Figure 1. Positions of the MMS1 spacecraft located between the magnetopause (black dashed line) and the bow shock (black dotted-dashed line), both of which are calculated by a 3D empirical model (Chao et al. 2002). We plot the projections of the spacecraft, magnetopause, and bow shock position on the $z=0$ plane of the GSE coordinate system. All events occur near the bow shock, as calculated based on the upstream solar wind conditions. The bow shock of event 1 is plotted as a reference.

sub-electron scales. This analysis provides additional observational insight into the kinetic-scale energy cascading and dissipation processes. We study the difference of sub-ion-scale turbulence between a prenoon region and a postnoon region in the magnetosheath. For this analysis, we use measurements of the magnetosheath from the Magnetospheric MultiScale $(M M S)$ spacecraft.

\section{Data Analysis}

We conduct an observational analysis on seven events of magnetosheath turbulence measured with the MMS1 spacecraft. Among them, four events are downstream of a postnoon side (e.g., Gershman et al. 2014) bow shock configuration (events 1, 2,3 , and 4), while the other three events are downstream of a prenoon side bow shock configuration (events 5, 6, and 7). Figure 1 shows the positions of the MMS1 spacecraft for these events, and Table 1 exhibits the corresponding average solar wind conditions. Events on the upstream side of the prenoon regions correspond to slow solar wind, and events on the upstream side of postnoon regions correspond to fast solar wind. During these time intervals, burst mode data of some crucial physical quantities are available.

The magnetic field vectors are measured by the Fluxgate Magnetometer (Russell et al. 2016) and the Search-Coil Magnetometer (Le Contel et al. 2016). The three components of the electric field are measured by the Spin-Plane Double Probe (Lindqvist et al. 2016) and the Axial Double Probe (Ergun et al. 2016). We acquire the number density, velocity, and temperature of both ions and electrons from the Fast Plasma Investigation 


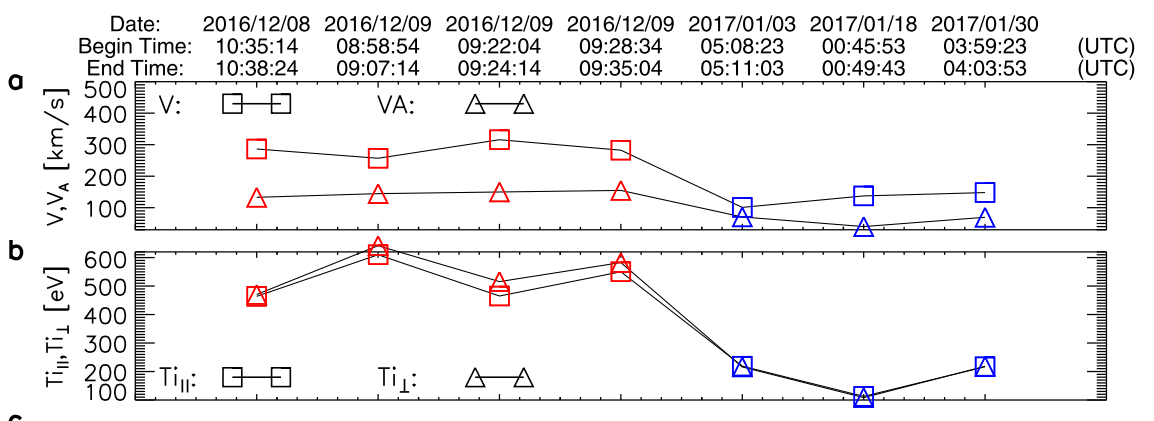

C

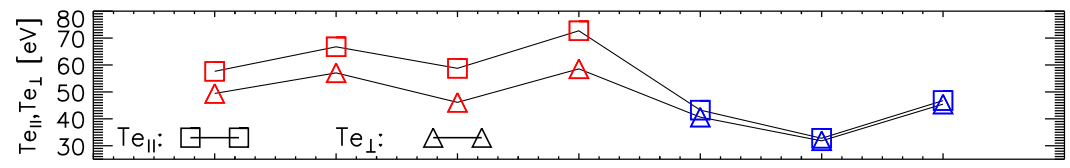

d

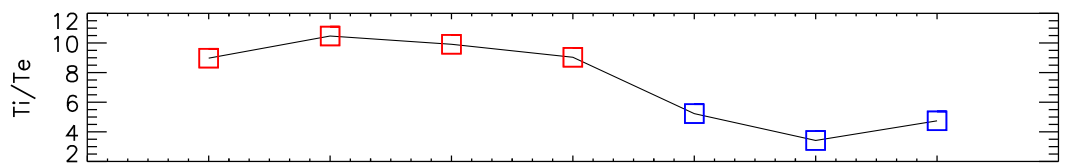

e

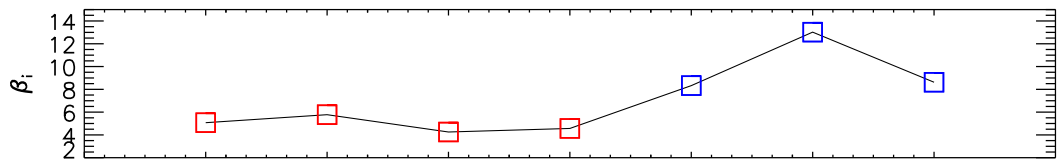

f
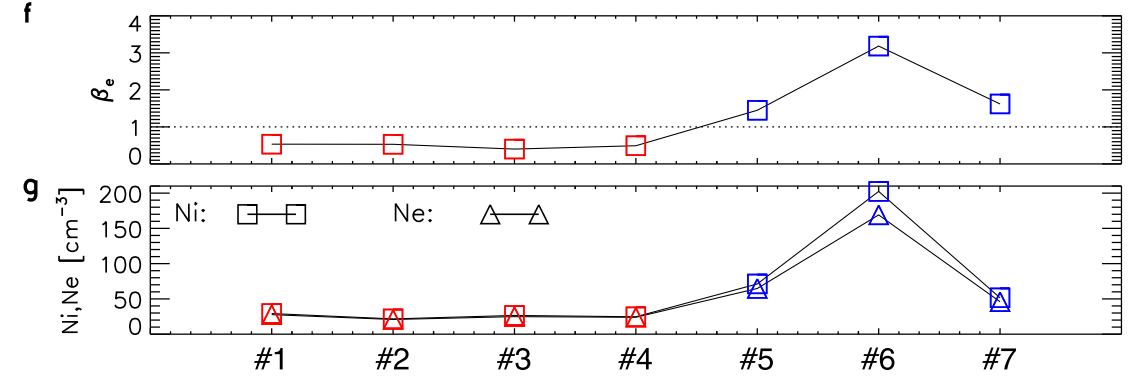

Figure 2. Average values of some basic plasma parameters for seven events: (a) bulk flow speed and Alfvén speed $\left(V_{\mathrm{A}}=B_{0} / \sqrt{\mu_{0} \rho_{i}}\right)$ ); ((b) and (c)) parallel and perpendicular temperatures of ions and electrons, respectively; (d) ratio of total ion to total electron temperature; (e) ion plasma beta $\left(\beta_{i}=n_{i} T_{i} /\left(B^{2} / 2 \mu_{0}\right)\right) ;(\mathrm{f})$ electron plasma beta $\left(\beta_{e}=n_{e} T_{e} /\left(B^{2} / 2 \mu_{0}\right)\right)$; and (g) ion and electron number density. Red and blue symbols represent events in the postnoon region and prenoon region, respectively.

Table 1

Parameters for the Upstream Solar Wind

\begin{tabular}{lcccccccc}
\hline \hline Event & $\mathrm{IMF}_{\mathrm{GSE}}(\mathrm{nT})$ & $\theta_{\mathrm{nB}}\left(^{\circ}\right)$ & $V_{\mathrm{sw}}\left(\mathrm{km} \mathrm{s}^{-1}\right)$ & $n_{p}\left(\mathrm{~cm}^{-3}\right)$ & $T_{p}(\mathrm{MK})$ & $\Delta R(\mathrm{Re})$ & Region & Shock Type \\
\hline 1 & {$[6.48,-5.35,2.47]$} & 52.20 & 564.46 & 5.56 & 0.38 & 0.10 & Postnoon \\
2 & {$[7.24,-3.03,2.97]$} & 36.49 & 612.92 & 4.66 & 0.29 & 0.14 & Oblique \\
3 & {$[6.96,-4.77,0.65]$} & 43.77 & 602.48 & 4.62 & 0.25 & 0.16 & Postnoon & Oblique \\
4 & {$[6.97,-4.48,1.37]$} & 42.76 & 594.32 & 4.49 & 0.23 & 0.26 & Postnoon & Oblique \\
5 & {$[-3.44,3.85,0.38]$} & 138.60 & 372.27 & 12.24 & 0.04 & 0.10 & Oblique \\
6 & {$[-3.96,1.17,0.45]$} & 171.67 & 308.38 & 34.09 & 0.02 & 0.83 & Prenoon & Oblique \\
7 & {$[-4.28,0.17,-1.30]$} & 158.55 & 400.81 & 7.73 & 0.04 & 0.86 & Prenoon & Quasi-parallel \\
& & & & & & Quasi-parallel \\
\hline
\end{tabular}

Note. From left to right: the event number, the direction of interplanetary magnetic field (IMF) in GSE coordinates, the shock angle, the solar wind speed, the proton density, the proton temperature, the geocentric radial distance between the spacecraft and the bow shock, the spacecraft's location and the shock type. (The solar wind basic parameters are obtained from https://omniweb.gsfc.nasa.gov.)

(Pollock et al. 2016), using data version 3.1. The resolution of burst mode data is $7.8 \mathrm{~ms}$ for the magnetic field, $0.12 \mathrm{~ms}$ for the electric field, $150 \mathrm{~ms}$ for ion moments, and $30 \mathrm{~ms}$ for electron moments. We illustrate the average values of some typical plasma parameters in Figure 2. This figure clearly shows that the plasma properties crucially depend on the position of the spacecraft. Our events downstream of a postnoon bow shock have the following approximate average plasma conditions: $T_{i}$ ranges from 450 to
$650 \mathrm{eV}, T_{e}$ ranges from 50 to $65 \mathrm{eV}, T_{i} / T_{e} \sim 10, \beta_{i} \sim 5$, $\beta_{e} \sim 0.5, n_{i} \sim n_{e} \sim 25 \mathrm{~cm}^{-3}$ and $v_{i} \sim v_{e} \sim 180 \mathrm{~km} \mathrm{~s}^{-1}\left(v_{i}\right.$ and $v_{e}$ are the ion and electron velocities). Our events downstream of a prenoon bow shock have the following approximate plasma conditions: $T_{i}$ ranges from 100 to $250 \mathrm{eV}, T_{e}$ ranges from 30 to $45 \mathrm{eV}, T_{i} / T_{e} \sim 4, \quad \beta_{i} \sim 10, \quad \beta_{e} \sim 2.0, \quad n_{i} \sim n_{e} \sim 50 \mathrm{~cm}^{-3}$, and $v_{i} \sim v_{e} \sim 130 \mathrm{~km} \mathrm{~s}^{-1}$. All of these events present a weak anisotropy with $0.5<\frac{T_{i, \perp}}{T_{i, \|}}, \frac{T_{e, \perp}}{T_{e, \|}}<1.5, T_{i, \perp}>T_{i, \|}$, and $T_{e, \perp}<$ 

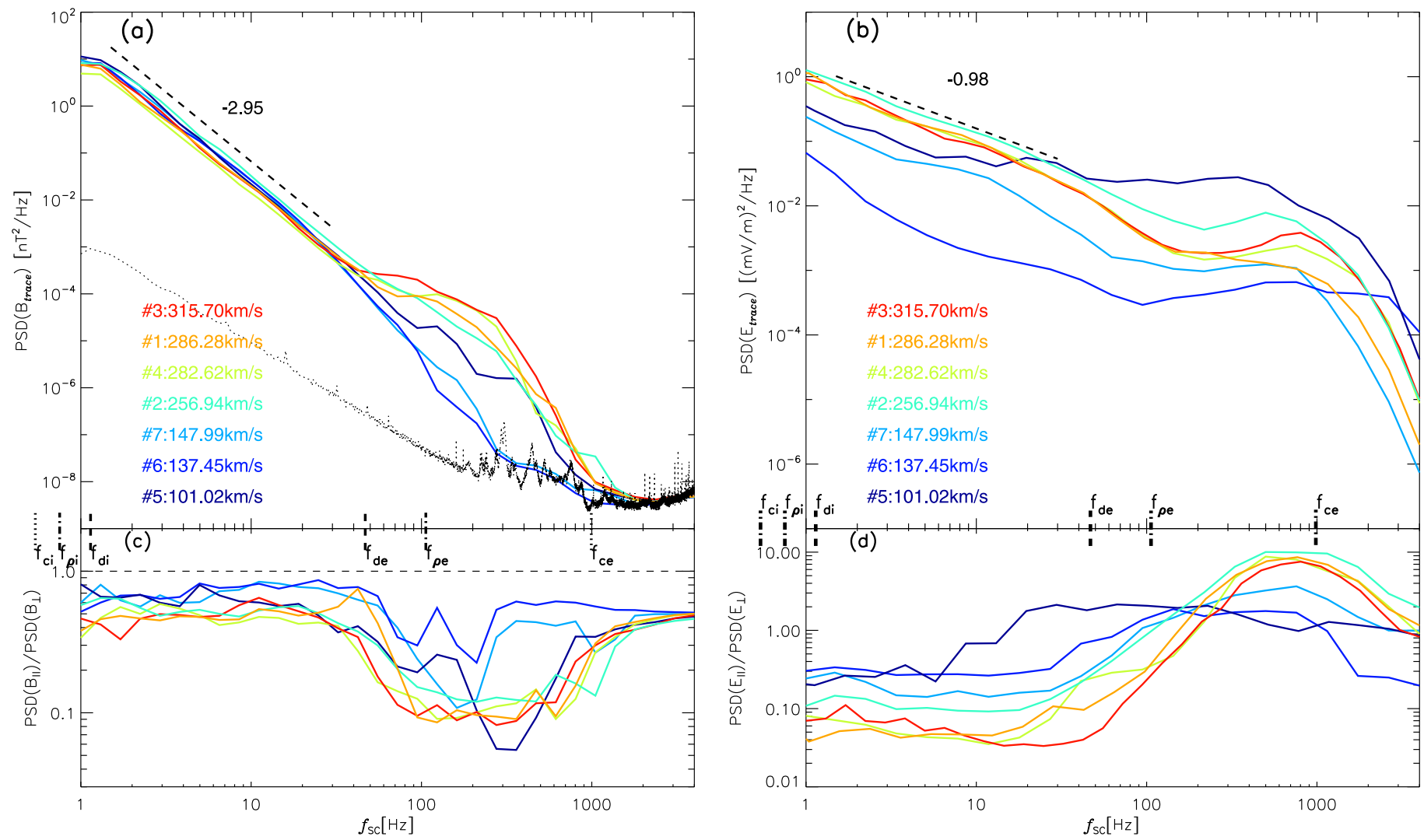

Figure 3. (a) Trace power spectral density (PSD) of the magnetic field. We fit the spectra of the seven events and then take the average (black dashed line). (b) Trace power spectral density (PSD) of the electric field. We fit the spectra of six events, excluding event 5 due to its large deviation from the other events, and then take the average (black dashed line). (c) Scale-dependent magnetic compressibility. (d) Ratio of $\operatorname{PSD}\left(E_{\|}\right)$to $\operatorname{PSD}\left(E_{\perp}\right)$. The dotted line is the PSD of the magnetic field in the lobe region (2016 July 6 08:18:44-08:20:43), which serves as a proxy of the inflight noise level.

$T_{e, \|}$ on average $\left(T_{s, \perp}\right.$ and $T_{s, \|}$ are the perpendicular and parallel temperatures of species $s$ with respect to the local background magnetic field, respectively). The discrepancy between the ion and electron densities in event 6 suggests that the electrostatic analyzers for electrons potentially saturated during this event. The only plasma moment we use in our analysis is the ion velocity in order to derive the convective electric field, so that this artificial density discrepancy does not impact our conclusions.

Figures 3(a) and (b) show the trace PSD of the magnetic field and the electric field. We eliminate the convective electric field (i.e., $\left.\boldsymbol{E}^{\prime}=\boldsymbol{E}+\boldsymbol{v}_{\boldsymbol{i}} \times \boldsymbol{B}\right)$. We see from panel (a) that the magnetic field spectra $\left(\operatorname{PSD}\left(B_{\text {trace }}\right)\right)$ at $f \in[1,30] \mathrm{Hz}$ have the same power level and spectral index (the average fitted spectral index $\alpha_{B}$ is -2.95). The frequency range between 1 and $30 \mathrm{~Hz}$ lies between ion scales and electron scales for all events according to Taylor's hypothesis (for its validity, see Chhiber et al. 2018). We justify the applicability of Taylor's hypothesis in two ways: the second-order structure function calculated from single-spacecraft data after transforming from the temporal domain to the spatial domain with Taylor's hypothesis is comparable to the spatial second-order structure function directly measured with six spacecraft separations. The applicability is also justified through the linear relation of the frequency in the spacecraft frame with the wavenumber calculated in our quantitative analysis below. Beyond $30 \mathrm{~Hz}$, the spectra begin to bifurcate and then converge again when reaching sub-electron scales at $\sim 1 \mathrm{kHz}$. The $\operatorname{PSD}\left(B_{\text {trace }}\right)$ segments for events on the prenoon-side downstream of the bow shock at frequencies greater than $30 \mathrm{~Hz}$ have similar scalings as their counterpart of PSD segments at frequencies between 1 and $30 \mathrm{~Hz}$. The $\operatorname{PSD}\left(B_{\text {trace }}\right)$ in the postnoon-side shock configuration shows a clear enhancement at frequencies between 30 and $300 \mathrm{~Hz}$, which is consistent with an enhanced population of whistler waves. We present the details of our wave diagnosis in the following section. $\operatorname{All} \operatorname{PSD}\left(B_{\text {trace }}\right)$ curves nearly aggregate at about $1 \mathrm{kHz}$. At $f>1 \mathrm{kHz}$, the spectra flatten, which is potentially attributed to instrument noise.

Figure 3(b) shows the trace PSD of the electric field $\left(\operatorname{PSD}\left(E_{\text {trace }}\right)\right)$. The difference between the measurements makes a universal analysis more difficult. The average fitted spectral index $\left(\alpha_{E}\right)$ is -0.98 at frequencies between 1 and $30 \mathrm{~Hz}$, which satisfies $\alpha_{E}=\alpha_{B}-2$, as suggested by the spectral scaling relation $\delta E^{2} \sim k^{2} \delta B^{2}$. This relation follows from the generalized Ohm's law with nonideal terms (e.g., Hall term: $J \times B / n e$ and pressure gradient term: $-\nabla p / n e$ ) (Matteini et al. 2017). Kinetic Alfvén waves exhibit this behavior because the coherent ion motion adjusts to the potential component of the electric field resulting in a Boltzmann-like response with $\delta n \propto \phi$ and $\delta n \propto \delta B$ (Chen \& Boldyrev 2017; Breuillard et al. 2018). Moreover, KAWs possess small magnetic compressibility $\left(\delta B_{\|}^{2} / \delta B_{\perp}^{2}<1\right)$ (He et al. 2012; Salem et al. 2012), which is in agreement with our observations (panel (c)). Therefore, we suggest that KAWs dominate this frequency range. We do not identify an enhancement in the frequency range between 30 and $300 \mathrm{~Hz}$ as in the magnetic spectrum. At around $800 \mathrm{~Hz}$, however, the spectra show a weak and wide bulge, which, as we discuss in the following paragraph, is a feature consistent 
with the presence of electrostatic ion acoustic waves. Above $1 \mathrm{kHz}$, noise effects lead to a rapid fall in the spectra.

The lower sensitivity of the electric field antenna along the spin axis may not severely deteriorate the data quality of the parallel electric field measurements, since the parallel direction is often oblique to the spin axis in our study. The spin frequency of $M M S$, which is around $0.05 \mathrm{~Hz}$, is below the frequency range of our study. Besides, the harmonic spin tones are not present in our PSD analysis of the electric field, suggesting that the wake effect may not be significant in the events we study.

We show the magnetic compressibility, a quantity related to magnetic variance anisotropy, in Figure 3(c). In the event presented by Chen \& Boldyrev (2017), the fluctuations transition at $k d_{e}>1$ to the IKAW regime, marked by a change of $\delta B_{\|}^{2} / \delta B_{\perp}^{2}$ from $1 /\left(1+2 / \beta_{i}\right)$ to 1 . In our events, the kinetic Alfvén waves have a magnetic compressibility slightly below $1 /\left(1+2 / \beta_{i}\right)$. We interpret this lower magnetic compressibility as a consequence of the presence of whistler waves (as confirmed in the following part of our study). We show the ratio of the squared parallel electric field to the squared perpendicular electric field fluctuations in the same frequency range in panel (d). The parallel and perpendicular fluctuations are calculated with respect to the scale-dependent local mean background magnetic field (Podesta 2009).

In order to understand the nature of the fluctuations at subelectron scales in the magnetosheath, we diagnose the fluctuations by applying the singular value decomposition method (Santolík et al. 2003) to the wavelet time-period spectrum. We select two representative events: one event with spectral enhancement (event 3) and one event without spectral enhancement (event 7) as shown in Figure 4. We find from panel (a1) that the PSD enhancement frequently emerges at around $100 \mathrm{~Hz}$ for some discrete time intervals. The enhancement mainly occurs in the perpendicular magnetic fluctuations, also seen as a depression of $\operatorname{PSD}\left(B_{\|}\right) / \operatorname{PSD}\left(B_{\perp}\right)$ in panel (b1). Some whistler-wave features stand out at six time intervals (i.e., $22: 30-22: 35 ; 22: 43-22: 53 ; 22: 55-23: 05 ; 23: 08-23: 18$; $23: 20-23: 35 ; 23: 40-23: 45$ ) at timescales of $0.001-0.02 \mathrm{~s}$ : the propagation direction of magnetic fluctuations is quasi-parallel relative to the local mean magnetic field $B_{0, \text { local }}$ (panel (c1)); the magnetic fluctuations are nearly perpendicular to the local mean magnetic field $B_{0, \text { local }}$ in polarization (panel (d1)); and the right-hand circular polarization of the magnetic fluctuations (panels (e1) and (f1)) and the electric fluctuations (panel (g1)). From the above, we suggest that this spectral enhancement is caused by quasi-parallel propagating, right-hand polarized whistler-mode waves. The events without enhancements, as shown in the right panels of Figure 4, do not show these signals. The weaker signal of electric field right-hand polarization, as compared with the signal of the magnetic field, is probably due to the electrostatic field of KAWs, which exists in addition to the inductive electric field of the whistler waves. We see from Figure 2 that, for all shown events in the magnetosheath, the electron temperature is approximately isotropic, which contradicts the scenario of whistler-wave generation through the whistler anisotropy instability. We also study the 12-level heat flux data provided by $M M S$ to investigate the possibility of whistler-wave generation by heat flux instabilities and find that the heat flux $Q_{e}$ is far below the threshold of linear instability, $Q_{e} / Q_{\max }=1 / \beta_{e}^{0.8}$ (Gary et al. 1999; Lacombe et al. 2014), where $Q_{\max }=(3 / 2) n_{e} k_{\mathrm{B}} T_{e} V_{\mathrm{th}, e \|}$
$\left(V_{\text {th }, e \|}=\sqrt{k_{\mathrm{B}} T_{e \|} / m_{e}}\right)$. This threshold applies to $0.10 \leqslant$ $\beta_{e} \leqslant 5.0$ and all observed $\beta_{e}$ fall within this range in our events. These observations of nonthermal particle properties suggest that the observed whistler waves are created by a nonlinear process, which requires further study.

Figure 5 presents signatures of ion acoustic waves occurring in event 5 . We identify two types of signals in the time-period spectrograms for the PSDs of $E_{\|}$and $E_{\perp}$ : the first type shows a festoon-shape of the PSD extending from greater to smaller periods (Gallagher 1985), for example, the signal occurring at around 05:09:02.700 on 2017 January 3. The second type is characterized by an isolated local maximum in the PSD, for example, the signal occurring at around 05:09:03.600 on 2017 January 3 . We suggest as a possible cause for the weaker enhanced power in the $E_{\perp}$ spectrum that the calculated $E_{\|}$and $E_{\perp}$ are not strictly aligned with the local mean-magnetic-field coordinate system due to the intense compressibility of the magnetosheath.

\section{Quantitative Analysis of the Wave Mode Composition}

In this section, we study the energy partition among different wave modes (whistler waves (WWs), kinetic Alfvén waves (KAWs), and ion acoustic waves (IAWs)). We assume a mixture of three wave modes, as suggested by our qualitative analysis in the previous section: quasi-parallel propagating slow modes $\left(\theta_{k B}=1^{\circ}\right)$ to account for IAWs at small scales, quasi-perpendicular propagating Alfvén/KAW modes $\left(\theta_{k B}=89^{\circ}\right)$ at small scales, and quasi-parallel propagating fast modes $\left(\theta_{k B}=5^{\circ}\right)$ for WWs at small scales. We calculate the dispersion relations and polarization relations for these three modes assuming the plasma conditions of event 3 with $\beta_{i}=4.26, \beta_{e}=0.40, d_{i}=44.07 \mathrm{~km}$, $\omega_{c i}=3.40 \mathrm{rad} \mathrm{s}^{-1}$ in the framework of two-fluid plasma theory (Zhao 2015). We transform the polarization relations from wavenumber space to the frequency domain in the spacecraft reference frame according to $\omega_{\mathrm{sc}}=\omega_{\mathrm{pl} .}+\boldsymbol{k} \cdot \boldsymbol{v}$, where $\omega_{\mathrm{sc}}$ is frequency in the spacecraft reference frame, $\omega_{\text {pl. }}$ is frequency in the plasma frame and $v$ is bulk plasma velocity, with the assumption that $\boldsymbol{k} \| \boldsymbol{v}$. We find that $\omega_{\text {sc }}$ follows a linear relationship with the wavenumber, which also justifies our use of Taylor's hypothesis. Figure 6 shows the polarization curves for the different wave modes. The observed $\operatorname{PSD}\left(B_{\|}\right) / \operatorname{PSD}\left(B_{\perp}\right)$ and $\operatorname{PSD}\left(E_{\|}\right) / \operatorname{PSD}\left(E_{\perp}\right)$ of event 3 are also displayed in the same figure.

We first define the ratios

$$
\alpha_{\mathrm{ww} / \mathrm{kaw}}^{\delta B_{1}^{2}}=\frac{\delta B_{\perp, \text { whistler }}^{2}}{\delta B_{\perp, \mathrm{kaw}}^{2}}
$$

and

$$
\alpha_{\text {iaw } / \text { kaw }}^{\delta B^{2}}=\frac{\delta B_{\perp, \text { iaw }}^{2}}{\delta B_{\perp, \text { kaw }}^{2}} .
$$

The two-fluid calculation then provides us with the polarization ratios

$$
\begin{gathered}
R_{B, \text { kaw }}=\frac{\delta B_{\|, \text {kaw }}^{2}}{\delta B_{\perp, \text { kaw }}^{2}}, \\
R_{B, \text { whistler }}=\frac{\delta B_{\|, \text {whistler }}^{2}}{\delta B_{\perp, \text { whistler }}^{2}}
\end{gathered}
$$



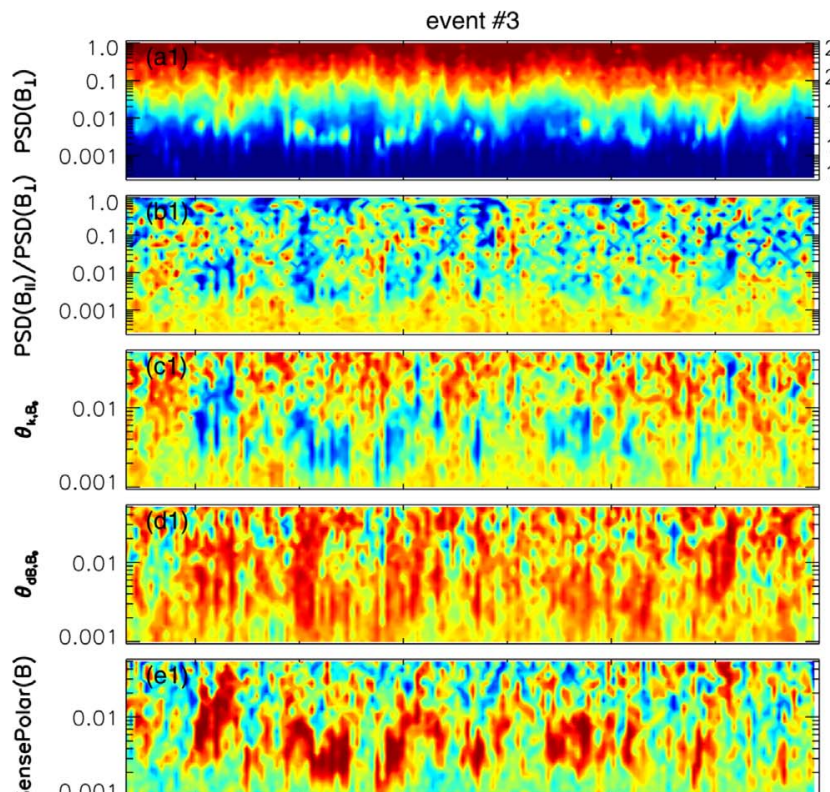

ஸे 0.001

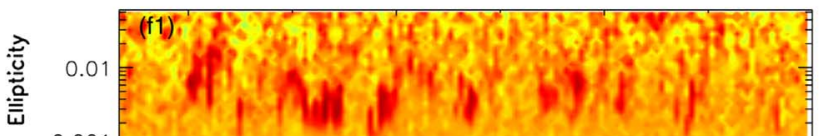

0.001

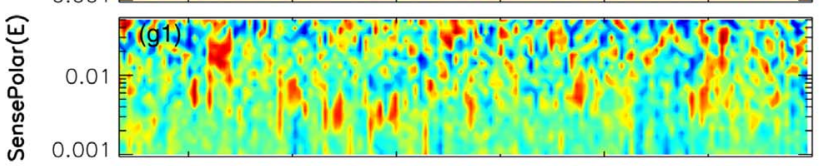

09:22:30

09:23:00

09:23:30

09:24:00
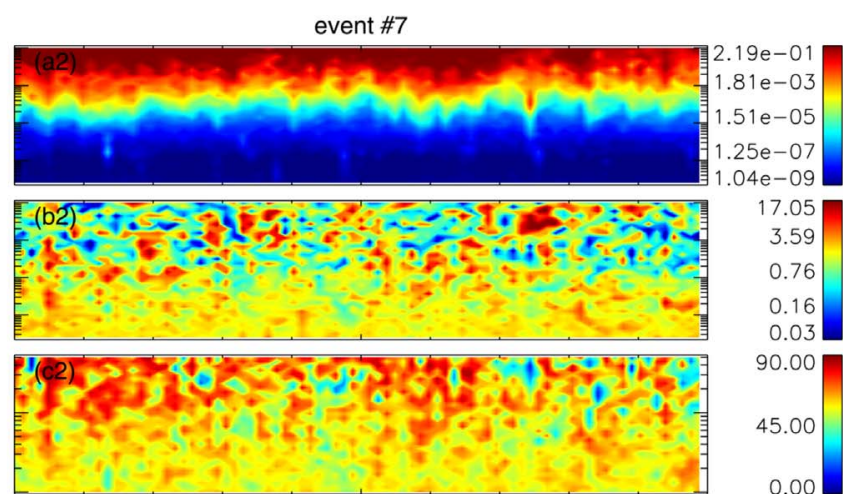

90.00
45.00

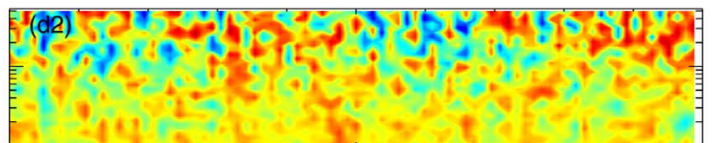

0.00

1.00
0.00
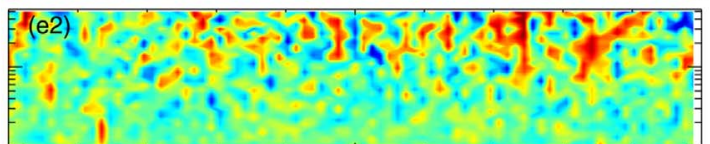

$-1.00$

0.00
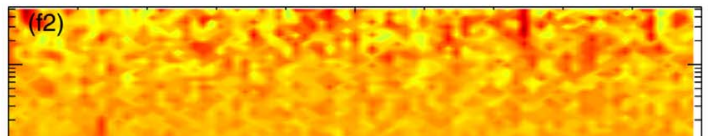

-1.00
1.00
0.00
-1.00

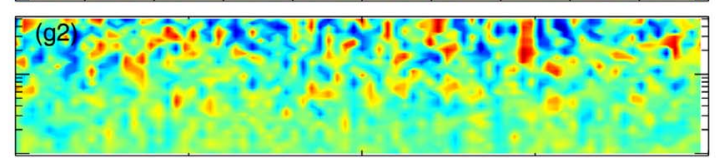

04:00:30

04:01:00
3.59

0.76

0.16

0.03

90.00

45.00

0.00

90.00

45.00

0.00

0.00

$-1.00$

0.00

$-1.00$

1.00

0.00

$-1.00$

Figure 4. Left panels show event 3 with an enhancement in the magnetic spectrum (signature of whistler waves). Right panels show event 7 without such an enhancement in $\operatorname{PSD}\left(B_{\text {trace }}\right)$. (a) Time-period spectrogram of $\operatorname{PSD}\left(B_{\perp}\right)$. (b) Time-period spectrogram of $\delta B_{\|}^{2} / \delta B_{\perp}^{2}$. (c) Time-period spectrogram of the angle between the wavevector of the electromagnetic fluctuations and the local mean magnetic field direction, $\theta_{k, B_{0}}$. . (d) Time-period spectrogram of the angle of the local magnetic fluctuations with respect to the local mean magnetic field, $\theta_{d B, B_{0, \text { local }}}$. (e) Time-period spectrogram of the sense of polarization for the magnetic fluctuations around the local mean magnetic field direction ( -1 : left-hand polarization; +1: right-hand polarization); (f) time-period spectrogram of the ellipticity of the magnetic fluctuations with the value near unity representing circular polarization. (g) Time-period spectrogram of the sense of polarization for the electric field fluctuations around the local mean magnetic field direction. The convective electric field due to the ion mean bulk flow has been removed.

$$
\begin{aligned}
& R_{B, \text { iaw }}=\frac{\delta B_{\| \text {,iaw }}^{2}}{\delta B_{\perp, \text { iaw }}^{2}} \\
& R_{E, \text { kaw }}=\frac{\delta E_{\| \text {,kaw }}^{2}}{\delta E_{\perp, \text { kaw }}^{2}}, \\
& R_{E \text {,whistler }}=\frac{\delta E_{\|, \text {whistler }}^{2}}{\delta E_{\perp, \text { whistler }}^{2}}, \\
& R_{E \text {,iaw }}=\frac{\delta E_{\| \text {,iaw }}^{2}}{\delta E_{\perp, \text { iaw }}^{2}} \\
& \beta_{E, \text { kaw }}=\frac{\delta E_{\perp, \mathrm{kaw}}^{2}}{\delta B_{\perp, \mathrm{kaw}}^{2}}, \\
& \beta_{E \text {,whistler }}=\frac{\delta E_{\perp, \text { whistler }}^{2}}{\delta B_{\perp, \text { whistler }}^{2}}, \\
& \beta_{E \text {,iaw }}=\frac{\delta E_{\perp \text {,iaw }}^{2}}{\delta B_{\perp, \text { iaw }}^{2}}
\end{aligned}
$$

for the three wave modes under consideration. $\delta B_{\perp(\|), x}^{2}$ and $\delta E_{\perp(\|), x}^{2}$ are the contributions to the perpendicular (parallel) fluctuating magnetic and electric fields relative to the background magnetic field from mode $x$.

We regard the total fluctuations as a combination of fluctuations from these three modes. Then, we find for the predicted fluctuations measured by the spacecraft:

$$
\begin{aligned}
& \delta B_{\|, \mathrm{sc}}^{2}=\delta B_{\perp, \mathrm{kaw}}^{2}\left(R_{B, \mathrm{kaw}}+\alpha_{\mathrm{ww} / \mathrm{kaw}}^{\delta B_{\perp}^{2}} \cdot R_{B, \text { whistler }}\right. \\
& \left.+\alpha_{\text {iaw } / \text { kaw }}^{\delta B^{2}} \cdot R_{B \text {,iaw }}\right) \\
& \delta B_{\perp, \mathrm{SC}}^{2}=\delta B_{\perp, \mathrm{kaw}}^{2}\left(1+\alpha_{\mathrm{ww} / \mathrm{kaw}}^{\delta B_{\perp}^{2}}+\alpha_{\text {iaw/kaw }}^{\delta B_{\perp}^{2}}\right) \\
& \delta E_{\|, \mathrm{sc}}^{2}=\delta E_{\perp, \mathrm{kaw}}^{2}\left(R_{E, \mathrm{kaw}}+\frac{\beta_{\mathrm{whistler}}}{\beta_{\text {kaw }}} \cdot \alpha_{\mathrm{ww} / \mathrm{kaw}}^{\delta B^{2}}\right. \\
& \left.\cdot R_{B, \text { whistler }}+\frac{\beta_{\text {iaw }}}{\beta_{\text {kaw }}} \cdot \alpha_{\text {iaw } / \text { kaw }}^{\delta B_{2}^{2}} \cdot R_{B, \text { iaw }}\right) \\
& \delta E_{\perp, \mathrm{sc}}^{2}=\delta E_{\perp, \mathrm{kaw}}^{2}\left(1+\frac{\beta_{\mathrm{whistler}}}{\beta_{\text {kaw }}} \cdot \alpha_{\mathrm{ww} / \text { kaw }}^{\delta B_{\perp}^{2}}+\frac{\beta_{\text {iaw }}}{\beta_{\text {kaw }}} \cdot \alpha_{\text {iaw } / \text { kaw }}^{\delta B^{2}}\right) .
\end{aligned}
$$




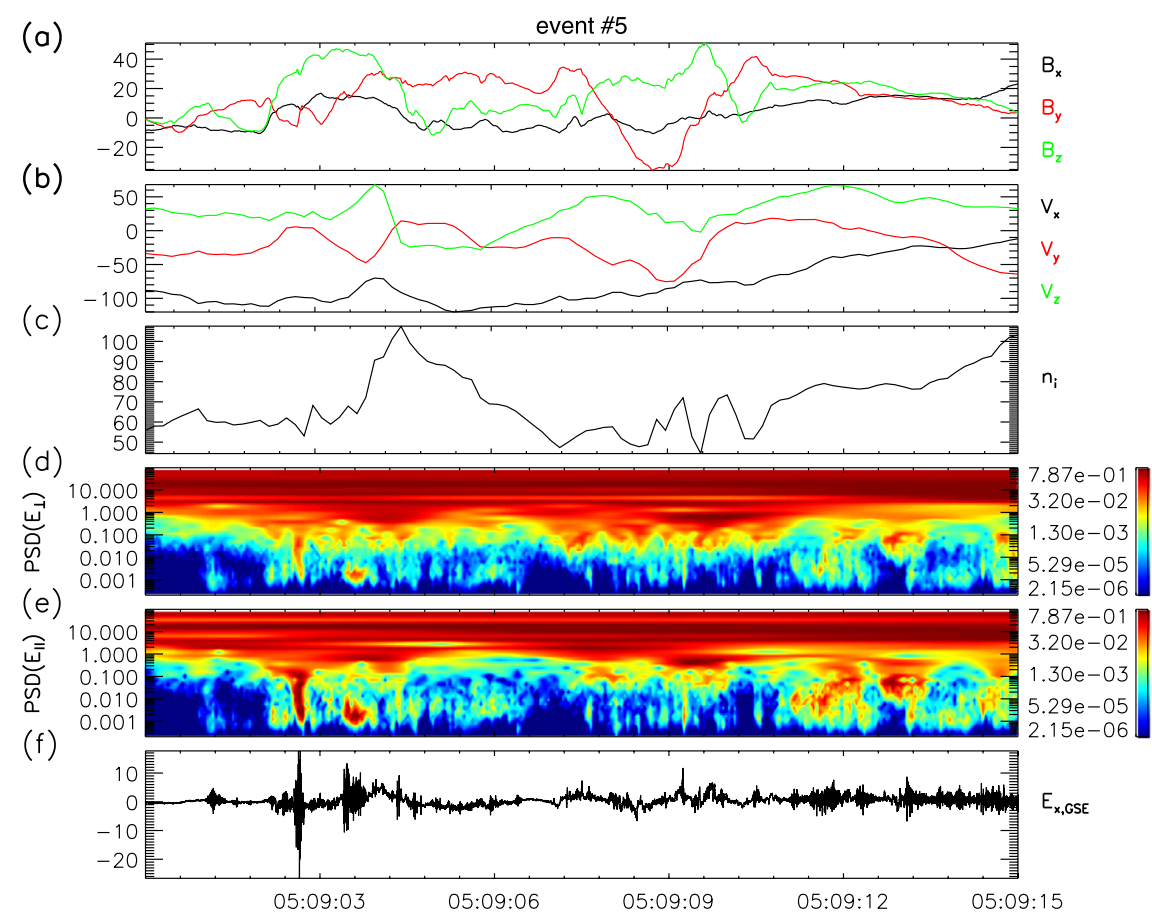

Figure 5. Signatures of two types of ion acoustic wave patterns in the time-period spectrogram during event 5. (a) Time series of $B x, B y$, and $B z$ in GSE coordinates. (b) Time series of $V x, V y$, and $V z$ in GSE coordinates; (c) time series of $n_{i}$ based on data from the FPI instrument. (d) Time-period spectrogram of the power spectral density of $E_{\perp}$. (e) Time-period spectrogram of the power spectral density of $E_{\|}$. (f) Time series of $E_{x, \text { GSE }}$ from EDP burst mode.

By dividing Equation (6) by (7) and Equation (8) by (9), we find

$$
\begin{aligned}
& {\left[\begin{array}{cc}
\left(R_{B, \text { whistler }}-\frac{\delta B_{\|, \mathrm{sc}}^{2}}{\delta B_{\perp, \mathrm{sc}}^{2}}\right) & \left(R_{B, \text { iaw }}-\frac{\delta B_{\|, \mathrm{sc}}^{2}}{\delta B_{\perp, \mathrm{sc}}^{2}}\right) \\
\frac{\beta_{\text {whistler }}}{\beta_{\text {kaw }}}\left(R_{E, \text { whistler }}-\frac{\delta E_{\|, \mathrm{sc}}^{2}}{\delta E_{\perp, \mathrm{sc}}^{2}}\right) & \frac{\beta_{\text {iaw }}}{\beta_{\text {kaw }}}\left(R_{E, \text { iaw }}-\frac{\delta E_{\|, \mathrm{sc}}^{2}}{\delta E_{\perp, \mathrm{sc}}^{2}}\right)
\end{array}\right]} \\
& \times\left[\begin{array}{l}
\alpha_{\mathrm{ww} / \mathrm{kaw}}^{\delta B^{2}} \\
\alpha_{\mathrm{iaw} / \mathrm{kaw}}^{\delta B^{2}}
\end{array}\right]=\left[\begin{array}{l}
\frac{\delta B_{\|, \mathrm{sc}}^{2}}{\delta B_{\perp, \mathrm{sc}}^{2}}-R_{B, \mathrm{kaw}} \\
\frac{\delta E_{\|, \mathrm{sc}}^{2}}{\delta E_{\perp, \mathrm{sc}}^{2}}-R_{E, \mathrm{kaw}}
\end{array}\right] .
\end{aligned}
$$

The solutions to Equation (10) are $\alpha_{\mathrm{ww} / \text { kaw }}^{\delta B_{\perp}^{2}}$ (the ratio of WW $\delta B_{\perp}^{2}$ to KAW $\delta B_{\perp}^{2}$ ) and $\alpha_{\text {iaw } / \text { kaw }}^{\delta B^{2}}$ (the ratio of IAW $\delta B_{\perp}^{2}$ to KAW $\delta B_{\perp}^{2}$ ). These quantities apply to all wavenumbers in principle, and we solve Equation (10) at $k d_{i}$ from 0.1 to 4000 , which covers the wavenumber domain corresponding to the frequency domain we consider in this study. For the other fluctuating quantities (e.g., $\delta B_{\|}^{2}, \delta E_{\perp}^{2}, \delta E_{\|}^{2}$ ), our calculation follows the same procedure. We list the results of our calculation for six frequencies in Table 2. IAWs contribute mostly to $\delta E_{\|}^{2}$, while they make a rather small contribution to the other amplitude components. For $\delta E_{\|}^{2}$, the ratios of WWs to KAWs are greater than unity at frequencies below $30 \mathrm{~Hz}$ and less than unity at frequencies above $30 \mathrm{~Hz}$. $\delta E_{\perp}^{2}$ mainly stems from KAWs with $\alpha_{\mathrm{ww} / \mathrm{kaw}}^{\delta E_{1}^{2}}$ less than about 0.01 below $30 \mathrm{~Hz}$ and about $0.01 \sim$ 0.1 above $30 \mathrm{~Hz}$. Perpendicular magnetic fluctuations $\left(\delta B_{\perp}^{2}\right)$ of KAWs exist predominantly in the frequency range below $30 \mathrm{~Hz}$ $\left(0.1<\alpha_{\mathrm{ww} / \text { kaw }}^{\delta B_{\perp}^{2}}<1\right)$, whereas whistler waves account for the majority of $\delta B_{\perp}^{2}$ at frequencies above $30 \mathrm{~Hz}$ (roughly, $1<$ $\alpha_{\mathrm{ww} / \mathrm{kaw}}^{\delta B_{\perp}^{2}}<10$ ). Figure 7 shows the distribution of $\delta B_{\|}^{2} / \delta B_{\perp}^{2}$ and $\delta E_{\|}^{2} / \delta E_{\perp}^{2}$ in parameter space $\left(\alpha_{\text {iaw } / \text { kaw }}^{\delta B_{1}^{2}}, \alpha_{\mathrm{ww} / \text { kaw }}^{\delta B^{2}}\right)$ to illustrate the effect of the $\delta B_{\perp}^{2}$-ratios for different modes on the observable total polarization. We see that $\delta B_{\|}^{2} / \delta B_{\perp}^{2}$ is mostly sensitive to $\alpha_{\mathrm{ww} / \mathrm{kaw}}^{\delta B_{\perp}^{2}}$, while $\delta E_{\|}^{2} / \delta E_{\perp}^{2}$ is mostly sensitive to $\alpha_{\text {iaw } / \text { kaw }}^{\delta B_{\perp}^{2}}$ for all frequencies. Therefore, $\delta B_{\|}^{2} / \delta B_{\perp}^{2}$ is a reliable indicator for the existence of WWs, while $\delta E_{\|}^{2} / \delta E_{\perp}^{2}$ acts as an indicator for the existence of IAWs.

\section{Discussion and Conclusions}

We investigate the nature of turbulence from ion scales at about $1 \mathrm{~Hz}$ to sub-electron scales at about $1 \mathrm{kHz}$ in the magnetosheath based on seven events obtained from the MMSI spacecraft. Between ion scales and the electron inertial scale, the $\operatorname{PSD}\left(B_{\text {trace }}\right)$ measurements are approximately equal, which is consistent with observations in the solar wind (Bruno et al. 2014). At the electron inertial scale, the PSDs of different events bifurcate. Bruno et al. (2014) present an identical behavior of the magnetic PSD at sub-ion scales in the solar wind regardless of the flow speed. The equal behavior and amplitude of magnetic PSDs at sub-ion scales for various types of solar wind is attributed to more dissipation at sub-ion scales in high speed flows, leading to hotter protons and alpha particles. In our study, we find the same behavior in the magnetosheath. The transition of upstream solar-wind flows with different speeds, the dependence of the enhancement of turbulence on the flow speed, and the PSD behavior at sub-ion scales for different upstream conditions are interesting topics for future study. 

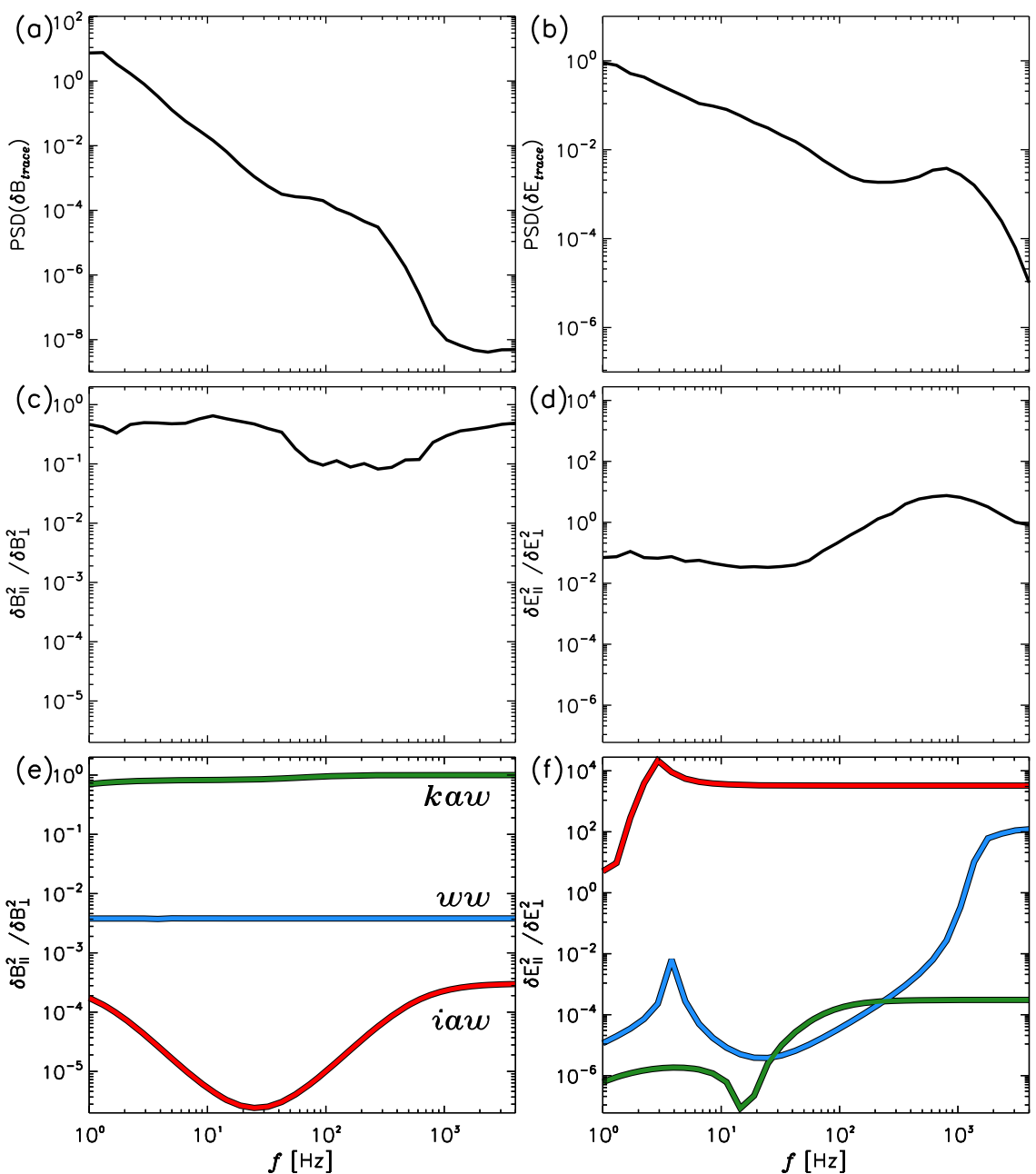

Figure 6. Panels (a) through (d) show observational results for event 3. Panels (a) and (b): trace power spectral densities of the magnetic field and the electric field. Panel (c): ratio $\operatorname{PSD}\left(\delta B_{\|}^{2}\right) / \operatorname{PSD}\left(\delta B_{\perp}^{2}\right)$. Panel (d): ratio of $\operatorname{PSD}\left(\delta E_{\|}^{2}\right) / \operatorname{PSD}\left(\delta E_{\perp}^{2}\right)$. Panels (e) and (f) show predictions for the magnetic field and electric field polarizations as functions of the Doppler-shifted frequency for kinetic Alfvén waves (kaw), whistler waves (ww), and ion acoustic waves (iaw) according to linearized two-fluid theory. We use the averaged plasma conditions of event 3 for the calculations shown in panels (e) and (f).

Table 2

Energy Ratios of the Field Amplitudes $\left(B_{\perp}, B_{\|}, E_{\perp}\right.$, and $\left.E_{\|}\right)$for KAWs, WWs, and IAWs at Frequencies from Ion to Sub-electron Scales

\begin{tabular}{|c|c|c|c|c|c|c|c|c|}
\hline$f(\mathrm{~Hz})$ & $\begin{array}{c}\alpha_{\mathrm{ww} / \mathrm{kaw}}^{2} \\
\alpha^{\prime}\end{array}$ & $\begin{array}{c}\delta B_{\perp}^{2} \\
\alpha_{\text {iaw } / \text { kaw }}\end{array}$ & $\begin{array}{c}\delta B_{\|}^{2} \\
\alpha_{\mathrm{ww} / \text { kaw }}\end{array}$ & $\begin{array}{c}\delta B_{\|}^{2} \\
\alpha_{\text {iaw/kaw }}\end{array}$ & $\overline{\alpha_{\mathrm{ww} / \text { kaw }}^{\delta E_{\perp}^{2}}}$ & $\begin{array}{c}\delta E_{\perp}^{2} \\
\alpha_{\text {iaw/kaw }}\end{array}$ & $\begin{array}{c}\delta E_{\|}^{2} \\
\alpha_{\text {ww/kaw }}\end{array}$ & $\begin{array}{c}\delta E_{\|}^{2} \\
\alpha_{\text {iaw/kaw }}\end{array}$ \\
\hline 8.503 & 0.434 & $7.25 \mathrm{E}-07$ & 0.126 & $1.32 \mathrm{E}-05$ & 0.002 & $5.92 \mathrm{E}-12$ & 1.843 & $4.32 \mathrm{E}+04$ \\
\hline 11.111 & 0.276 & $3.18 \mathrm{E}-07$ & 0.065 & $1.13 \mathrm{E}-05$ & 0.001 & $1.75 \mathrm{E}-12$ & 0.879 & $6.64 \mathrm{E}+04$ \\
\hline 72.298 & 7.342 & $2.61 \mathrm{E}-08$ & 0.341 & $4.70 \mathrm{E}-05$ & 0.030 & $1.68 \mathrm{E}-13$ & 0.058 & $1.51 \mathrm{E}+03$ \\
\hline 94.476 & 9.297 & $2.87 \mathrm{E}-08$ & 0.314 & $8.14 \mathrm{E}-05$ & 0.038 & $2.77 \mathrm{E}-13$ & 0.063 & $1.73 \mathrm{E}+03$ \\
\hline 803.317 & 3.391 & $4.60 \mathrm{E}-08$ & 0.003 & $2.31 \mathrm{E}-03$ & 0.013 & $9.25 \mathrm{E}-12$ & 0.286 & $2.51 \mathrm{E}+04$ \\
\hline 1049.742 & 2.367 & $5.75 \mathrm{E}-09$ & 0.001 & $2.01 \mathrm{E}-03$ & 0.009 & $1.35 \mathrm{E}-12$ & 0.638 & $2.18 \mathrm{E}+04$ \\
\hline
\end{tabular}

Note. $\delta B_{\perp}^{2}$ is dominated by KAWs at frequencies less than $30 \mathrm{~Hz}$ and by WWs at frequencies greater than $30 \mathrm{~Hz}$. $\delta B_{\|}^{2}$ and $\delta E_{\perp}^{2}$ primarily represent KAWs throughout the frequency range under consideration. $\delta E_{\|}^{2}$ is dominated by IAWs in the full frequency range.

Observations of magnetic and/or electric field polarization and waveforms are usually conducted (Zhang et al. 1998; Breneman et al. 2010; Lacombe et al. 2014) to identify the whistler mode. In this context, we highlight our finding that, in contrast to the magnetic spectral enhancement, the corresponding electric field signal does not stand out from the background turbulence PSD, showing no enhancement at the relevant scales. We identify two possible explanations for the different behavior in the magnetic and electric field signals: (1) $\operatorname{PSD}\left(E_{\text {trace }}\right)$ has contributions from both the whistler waves' electric field and the KAWs' electrostatic field; (2) alternatively, the steeper power-law profile of $\operatorname{PSD}(B)$ compared to the profile of $\operatorname{PSD}(E)$ can lead to a sampling effect. For example, when sampling along the magnetic field in a time series, the magnetic signal of quasi-parallel propagating waves with a given wavenumber can be stronger than the projected signal of obliquely propagating waves with greater wavenumber, while the electric signal of the same quasi-parallel propagating waves can be hidden by the enhanced projected signal of the same obliquely propagating waves with greater wavenumber. 


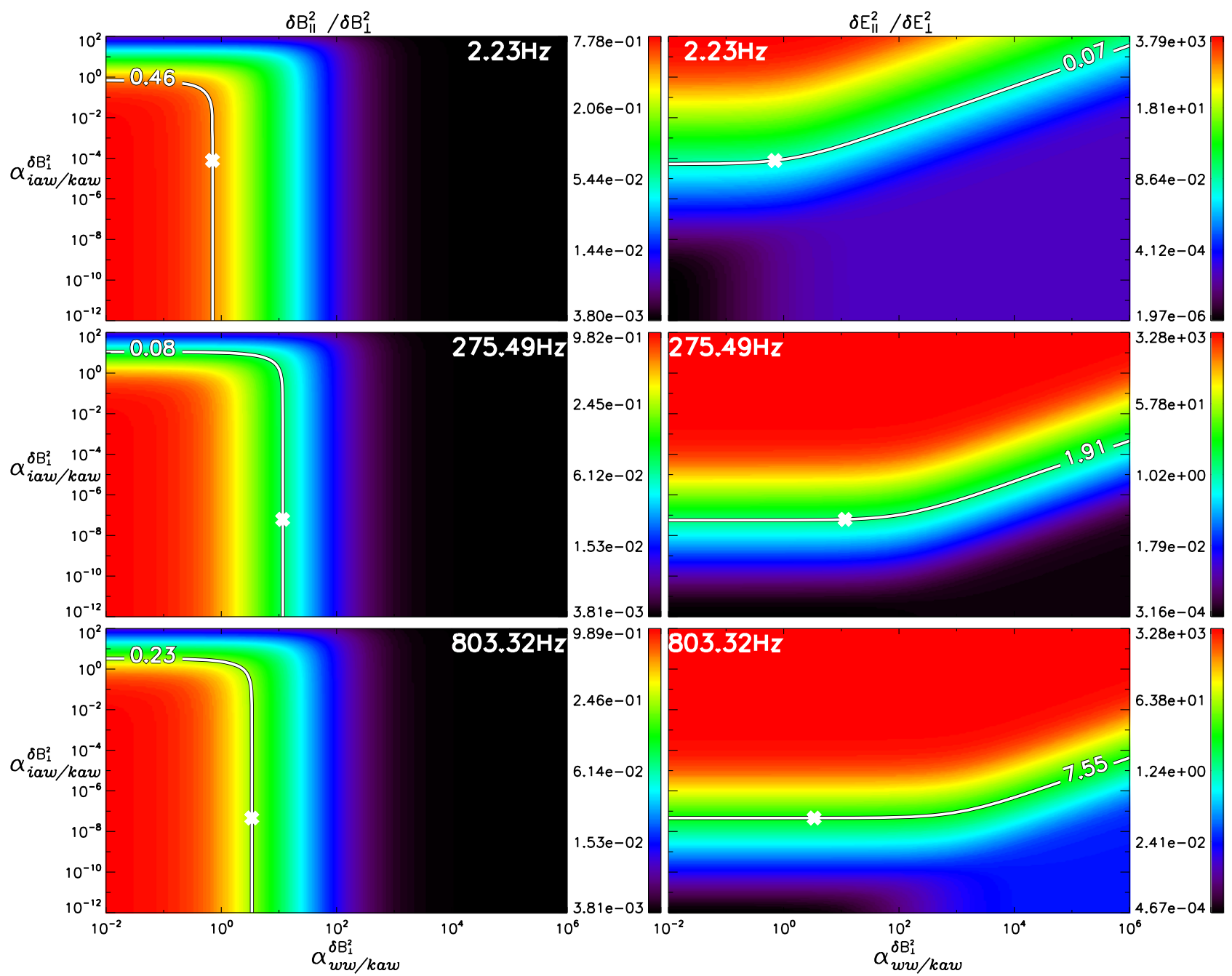

Figure 7. Distributions of $\delta B_{\|}^{2} / \delta B_{\perp}^{2}$ (color-coded on the left) and $\delta E_{\|}^{2} / \delta E_{\perp}^{2}$ (color-coded on the right) in the ( $\left.\alpha_{\text {ww } / \text { kaw }}^{\delta B_{\perp}^{2}}, \alpha_{\text {iaw/kaw }}^{\delta B_{\perp}^{2}}\right)$-plane as predicted from two-fluid theory. We show these distributions at three different frequencies in the spacecraft frame $(2.23,275.49,803.32 \mathrm{~Hz})$. We define the parameters $\alpha_{\mathrm{iaw}}^{\delta B_{\perp}^{2}} \mathrm{kaw}$ and $\alpha_{\mathrm{ww}}^{\delta B_{\perp}^{2}} / \mathrm{kaw}$ in Equations (1) and (2), The white lines indicate the contour that corresponds to the observed value from event 3.

In order to understand the underlying physics, we invoke a quantitative study of the difference between the $\operatorname{PSD}(B)$ and $\operatorname{PSD}(E)$ profiles by modeling a combination of wave modes (i.e., KAWs, WWs, and IAWs in our work) in the full range of frequencies from ion to electron scales. Our multi-component analysis successfully reproduces the observed PSDs of $B_{\perp}, B_{\|}$, $E_{\perp}, E_{\|}$. Based on the decomposition analysis of $\operatorname{PSD}\left(B_{\perp}\right)$, PSD $\left(B_{\|}\right), \operatorname{PSD}\left(E_{\perp}\right)$, and $\operatorname{PSD}\left(E_{\|}\right)$into contributions from these three wave modes, we learn that (1) $\operatorname{PSD}\left(B_{\perp}\right)$ is mainly related to KAWs and WWs at frequencies below and above $30 \mathrm{~Hz}$; (2) $\operatorname{PSD}\left(B_{\|}\right)$is primarily determined by KAWs throughout the investigated frequency range; (3) $\operatorname{PSD}\left(E_{\perp}\right)$ is mainly determined by KAWs with a secondary contribution from WWs in the full frequency range and (4) $\operatorname{PSD}\left(E_{\|}\right)$is mainly determined by IAWs with a secondary contribution from KAWs. These results also resolve the question as to why $\operatorname{PSD}\left(E_{\text {trace }}\right)$ does not show a spectral enhancement at the same frequencies at which $\operatorname{PSD}\left(B_{\text {trace }}\right)$ is enhanced.

Chen \& Boldyrev (2017) show that, occasionally, the turbulence in the magnetosheath is IKAW-like beyond the electron inertial scale. Our study demonstrates that the magnetosheath turbulence at sub-electron scales can consist of other types of electrostatic waves (e.g., ion acoustic waves) or electromagnetic waves (e.g., whistler waves) besides IKAWs, which may depend on plasma parameters such as $\beta_{i}$ and $T_{e} / T_{i}$. Therefore, the nature of magnetosheath turbulence at kinetic scales is complicated and diverse, as it can be composed of multiple components. Electrostatic modes beyond electron scales, which are likely IAWs, manifest as island-shapes in the time-period spectrogram.

The authors are grateful to the teams of the $M M S$ spacecraft for providing the data. The work at Peking University is supported by the National Natural Science Foundation of China (NSFC) under contracts 41574168, 41874200, and 41421003. D.V. is supported by the STFC Ernest Rutherford Fellowship ST/P003826/1. J.S.Z. is supported by NSFC 11673069 , 41531071. We thank O. Le Contel for useful suggestions about the noise level of the SCM.

\section{ORCID iDs}

Xingyu Zhu (1) https://orcid.org/0000-0002-1541-6397 Jiansen He (i) https://orcid.org/0000-0001-8179-417X Daniel Verscharen (1) https://orcid.org/0000-0002-0497-1096 Jinsong Zhao (1) https://orcid.org/0000-0002-3859-6394 


\section{References}

Alexandrova, O., Chen, C. H. K., Sorriso-Valvo, L., Horbury, T. S., \& Bale, S. D. 2013, SSRv, 178, 101

Alexandrova, O., Lacombe, C., Mangeney, A., Grappin, R., \& Maksimovic, M. 2012, ApJ, 760, 121

Alexandrova, O., Saur, J., Lacombe, C., et al. 2009, PhRvL, 103, 165003

Bale, S. D., Kellogg, P. J., Mozer, F. S., Horbury, T. S., \& Reme, H. 2005, PhRvL, 94, 215002

Belcher, J. W., \& Davis, L. 1971, JGR, 76, 3534

Breneman, A., Cattell, C., Schreiner, S., et al. 2010, JGRA, 115, 8104

Breuillard, H., Matteini, L., Argall, M., et al. 2018, ApJ, 859, 127

Bruno, R., \& Carbone, V. 2013, LRSP, 10, 2

Bruno, R., Trenchi, L., \& Telloni, D. 2014, ApJL, 793, 15

Chao, J. K., Wu, D. J., Lin, C.-H., et al. 2002, Space Weather Study Using Multipoint Techniques (Oxford: Elsevier)

Chen, C. H. K., Bale, S. D., Salem, C., \& Mozer, F. S. 2011, ApJL, 737, 41

Chen, C. H. K., \& Boldyrev, S. 2017, ApJ, 842, 122

Chhiber, R., Chasapis, A., Bandyopadhyay, R., et al. 2018, JGR, 123, 9941

Ergun, R. E., Tucker, S., Westfall, J., et al. 2016, SSRv, 199, 167

Fried, B. D., \& Gould, R. W. 1961, PhFl, 4, 139

Fried, B. D., \& Wong, A. Y. 1966, PhFl, 9, 1084

Fujimoto, K. 2014, GeoRL, 41, 2721

Gallagher, D. L. 1985, JGRA, 90, 1435

Gary, S. P. 1993, Theory of Space Plasma Microinstabilities (Cambridge: Cambridge Univ. Press)

Gary, S. P., Skoug, R. M., \& Daughton, W. 1999, PhPl, 6, 2607

Gary, S. P., \& Wang, J. 1996, JGRA, 101, 10749

Gershman, D. J., Raines, J. M., Slavin, J. A., et al. 2014, JGRA, 120, 4354

Gershman, D. J., Viñas, A. F., Dorelli, J. C., et al. 2018, PhPl, 25, 022303

Graham, D. B., Khotyaintsev, Y. V., Vaivads, A., \& André, M. 2016, JGRA, 121,3069

Gurnett, D. A., Frank, L. A., \& Lepping, R. P. 1976, JGR, 81, 6059

He, J., Pei, Z., Wang, L., et al. 2015, ApJ, 805, 176

He, J., Tu, C., Marsch, E., \& Yao, S. 2012, ApJL, 745, 8
He, J. S., Marsch, E., Tu, C. Y., et al. 2011, JGRA, 116, 6207

Hollweg, J. V., Kaghashvili, E. K., \& Chandran, B. D. G. 2013, ApJ, 769, 142

Howes, G. G., Bale, S. D., Klein, K. G., et al. 2012, ApJL, 753, L19

Huang, S. Y., Hadid, L. Z., Sahraoui, F., Yuan, Z. G., \& Deng, X. H. 2017 ApJL, 836, L10

Huang, S. Y., Sahraoui, F., Deng, X. H., et al. 2014, ApJL, 789, L28

Kolmogorov, A. N. 1941, DoSSR, 30, 301

Lacombe, C., Alexandrova, O., Matteini, L., et al. 2014, ApJ, 796, 5

Le Contel, O., Leroy, P., Roux, A., et al. 2016, SSRv, 199, 257

Lindqvist, P. -A., Olsson, G., Torbert, R. B., et al. 2016, SSRv, 199, 137

Matteini, L., Alexandrova, O., Chen, C. H. K., \& Lacombe, C. 2017, MNRAS, 466, 945

Narita, Y., Plaschke, F., Nakamura, R., et al. 2016, GeoRL, 43, 4774

Perschke, C., Narita, Y., Gary, S. P., Motschmann, U., \& Glassmeier, K. H. 2013, AnGeo, 31, 1949

Petrosyan, A., Balogh, A., Goldstein, M. L., et al. 2010, SSRv, 156, 135

Podesta, J. J. 2009, ApJ, 698, 986

Pollock, C., Moore, T., Jacques, A., et al. 2016, SSRv, 199, 331

Rodriguez, P., \& Gurnett, D. A. 1975, JGR, 80, 19

Russell, C. T., Anderson, B. J., Baumjohann, W., et al. 2016, SSRv, 199, 189

Sahraoui, F. 2003, JGR, 108, 1335

Sahraoui, F., Goldstein, M. L., Belmont, G., Canu, P., \& Rezeau, L. 2010 PhRvL, 105, 131101

Sahraoui, F., Goldstein, M. L., Robert, P., \& Khotyaintsev, Y. V. 2009, PhRvL, 102, 231102

Sahraoui, F., Huang, S. Y., Belmont, G., et al. 2013, ApJ, 777, 15

Salem, C. S., Howes, G. G., Sundkvist, D., et al. 2012, ApJL, 745, L9

Santolík, O., Parrot, M., \& Lefeuvre, F. 2003, RaSc, 38, 1010

Scarf, F. L., Frank, L. A., Ackerson, K. L., \& Lepping, R. P. 1974, GeoRL, 1, 189

Stawicki, O., Gary, S. P., \& Li, H. 2001, JGRA, 106, 8273

Tu, C.-Y., \& Marsch, E. 1995, SSRv, 73, 1

Yang, L., He, J., Tu, C., et al. 2017, ApJ, 836, 69

Yao, S., He, J. S., Marsch, E., et al. 2011, ApJ, 728, 146

Zhang, Y., Matsumoto, H., \& Kojima, H. 1998, JGRA, 103, 20529

Zhao, J. 2015, PhPl, 22, 042115 